\title{
IoT Based Infant Supervising System
}

\author{
Pearley Stanley, Lakshmi R, Sunanthini V, Gunaselvi Manohar, Sudha R
}

\begin{abstract}
In order to prevent infants from sudden infant death syndrome which is a serious problem among premature babies a wireless infant monitoring system has been designed. It measures every condition with respect to the baby such as position of sleeping, respiratory rate and baby's body temperature, humidity and heartbeat of an infant as well as its surrounding carbon monoxide concentration within the incubator. These parameters are considered to influence the sudden death of infants. They are measured and transmitted to the server which is located at a remote area with the help of WiFi. Alarms will be triggered if any of the monitored parameters is beyond the preset limits. The system overcomes the problems of the present system where the monitoring is done manually due to which there are risks of time delay of realizing or getting alerted in case of a serious issue with the child. The device can be easily integrated into cloud service so that a wireless communication can be established due to which parents can monitor their baby continuously from the home.

The project aims at proposing an automated system to measure the level of $\mathrm{Co} 2$ in the incubator which when high causes suffocation and also detect the occurrence of Fits in the baby whereby are death syndromes and prevent them by rapid actions. Along with this we measure other essential parameters and communicate these details continuously through IoT web technologies immediately to the required personnel.
\end{abstract}

Keywords: Monitoring, Internet of Things, Wireless, infant, death syndrome, sensing technology

\section{INTRODUCTION}

Sudden infant death syndrome (SIDS) is the sudden death of an infant without any previous viewable medical conditions. In US the death rate of infants have increased in recent times due to SIDS. However, it is very difficult to detect the cause of SIDS even after performing autopsy and examining the records of the infant. Extensive researches has revealed the fact that the body temperature, sleeping position of the baby and carbon monoxide exposure from0 smokers are the serious causes which leads to SIDS. According to K.Zhang [3], Meta- analysis can be carried out to measure the accumulated association of sudden infant death syndrome risk with both pre natal and post natal maternal smoking. By monitoring these parameters continuously an effective solution could be designed to detect the causes and intimate the parents and caretakers about the seriousness of SIDS so that preventive action

Revised Manuscript Received on 14 September, 2019.

Pearley Stanley, is with the Department of Electronics and Instrumentation Engineering, Easwari Engineering College, Tamil Nadu, India (E-mail:pearleysolace@gmail.com).

Lakshmi R, is with the Department of Electronics and Instrumentation Engineering, Easwari Engineering College, Tamil Nadu, India .

Sunanthini V, is with the Department of Electronics and Instrumentation Engineering, Easwari Engineering College, Tamil Nadu, India .

Gunaselvi Manohar, is with the Department of Electronics and Instrumentation Engineering, Easwari Engineering College, Tamil Nadu, India.

Sudha R, is with the Department of Electronics and Instrumentation Engineering, Easwari Engineering College, Tamil Nadu, India. could be taken without any delay. The wireless Infant Monitoring Device has been developed to address the need. The rapid advancement of wireless ommunication technology over the past decade has greatly improved the human life quality. As highlighted by G.D Clifford [9] wired technology can be cumbersome for patient monitoring and can restrict the behavior of the monitored patient introducing bias or artifacts. A.Darwish [5]stated that WBANs application in the medical field are composed of wearable and implantable sensors that can detect information from the human body. Also he stated that there is a difficulty in the monitoring devices that are not completely wearable where the wires are to connect many sensors. It has a significant impact on the design of modern wearable medical and healthcare devices. The ubiquitous wireless communication network allows wearable devices to be easily integrated into the cloud medical application servers. This enhances the healthcare quality through the real time surveillance and detection of abnormalities in patients that could otherwise be overlooked.

The risk factors of SIDS are often subtle, e.g. infants are more sensitive to suffocation in prone position that could be easily neglected by adults. Therefore, frequently monitoring those risk factors can minimize the occurrence of SIDS. Miniaturized wearable monitoring device is the perfect fit for the task.

\section{A. IoT (Internet of Things)}

It's modeling and simulation is carried out at the design stage before deployment of the network. Network simulators can be used to simulate IoT networks. Ambient intelligence and autonomous control are not part of the initial idea of the IoT. They do not necessarily require Internet structures, either. However, there is a need to integrate the concepts of the Internet of Things and autonomous control, with initial outcomes towards this direction considering objects as the driving force for autonomous IoT.

The Internet of Things (IoT) is the network of physical objects-devices, vehicles, building and other itemsembedded with electronics, software, sensors, and network connectivity that enables these objects to collect and exchange data. The IoT permits objects to be sensed and controlled remotely across existing network infrastructure, creating opportunities for further direct integration of the physical world into computer-based systems and resulting in enhanced performance, precision and financial profit; when IoT is augmented with sensors and actuators, the technology becomes an instance of the more general class of cyberphysical systems, which also incorporates technologies such as smart grids, smart homes, intelligent transport and smart 
cities. Each device is uniquely identifiable through its incorporated computing system but is able to inter-operate within the existing Internet infrastructure.

It also gives a clean atmosphere for the baby. It is a trolley with a mattress and on the top it is covered by a rigid clear plastic cover. The present day medical systems provide only the sterile condition for the baby's growth. It gives manual alert through nurses to doctors in case of any emergency conditions which causes time lag in reporting in case of immediate action. This leads to high death rate of the infant babies in the incubator.

\section{B. In existing system}

$\square \quad$ Very Complexity Hospitalization

$\square \quad$ system. Treatment may take long

$\square \quad$ time from Doctor. High death rate of infant baby.

\section{Proposed system Configuration}

This project's need is to eradicate the high number of deaths caused to the infants due to insufficient monitoring facilities in the hospitals. In the Internet of Things (IoT), devices collect and share data directly with each other. So collection, recording and analyzing of new data streams is much faster and accurate. After analyzing IoT provides all the possibilities across a range of Hospitals after dynamically calculating and report to the waiting Doctors. It is an effective systematic approach to solve the breathing problems caused in the incubator and also have a $24 * 7$ report of the baby's condition. It gives prompt online supervised conditions of the baby to the Doctors, Nurses and Parents through Internet of Things (IoT) Technology. Automatic alert to the person-in-charge and this safety system can also be used in hospitals and houses as well if needed.

\section{In Proposed system}

High Efficiency.

Easy Treatment Method by doctors on the spot.

Real-time Infant Monitoring even by parents.

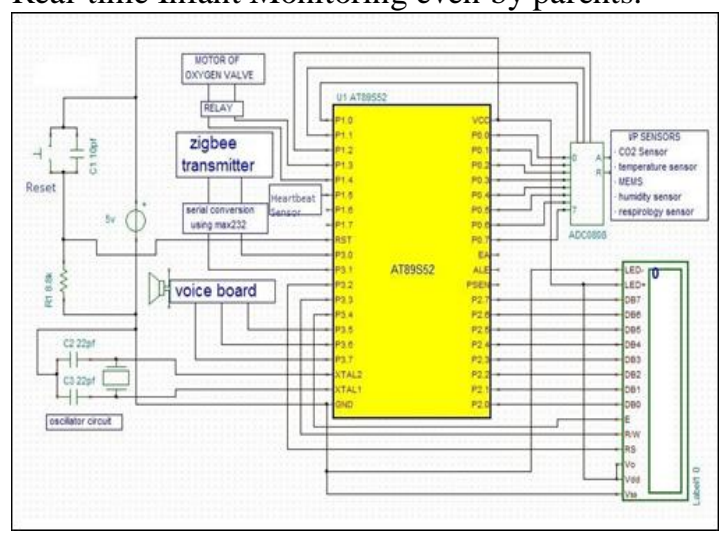

\section{E. MQ2 Sensors:}

These days, the incubators run on poor air filter faculties where the respired $\mathrm{CO} 2$ by the baby is not being properly released. There is an oxygen valve setup in our project where if the threshold is exceeded the motor runs to supply O2 until normal conditions are back. This reduces

Test Result : Cali Sensors

brated range $: 100 \mathrm{ppm}=1 \mathrm{Volt}$ suffocation to the baby ( one of the main reasons ofSIDS)
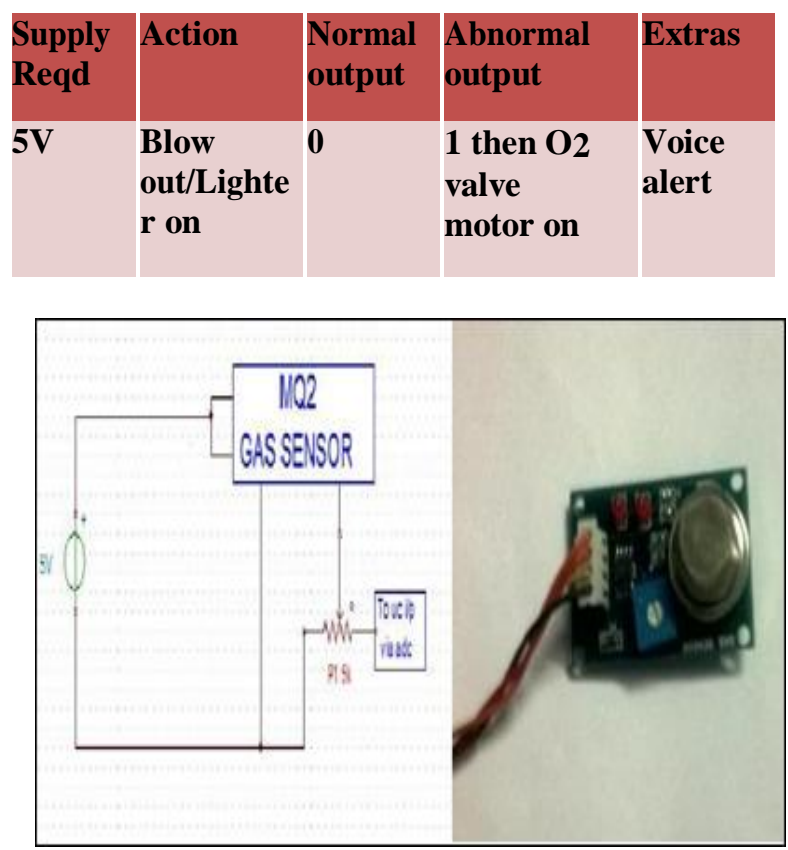

Fig 2.3 Temperature sensor

\section{F. Three axis accelerometer / MEMS sensor(adxl345)}

Sudden infant deaths are also caused if the Fits occur in babies and if they aren't responded quickly. In order to monitor if the baby is in sleeping/ active normal / irregular position, we have used 3 axis accelerometer to check and confirm its movements in all three axes. This chip can measure at rapid speeds up to $+16 \mathrm{~g}$. Runs at 5Volts supply. $\mathrm{Z}$ axis lies on the baby's chest wall

Test Result: calibrated range $:+3 g=3 \mathrm{~V}$

\begin{tabular}{|c|c|c|c|}
\hline & $\begin{array}{l}\text { Norma } \\
\text { I (+or- } \\
5)\end{array}$ & $\begin{array}{l}\text { Abnormal } \\
(+ \text { or }-5)\end{array}$ & Graphs \\
\hline Y axis & 84 & 49 & \multirow{3}{*}{$\begin{array}{l}\text { Straight line } \\
\text { when not } \\
\text { detecting. } \\
\text { Highly } \\
\text { erroneous } \\
\text { when } \\
\text { abnormal }\end{array}$} \\
\hline $\mathrm{Z}$ axis & 115 & 91 & \\
\hline Y axis & 84 & 49 & \\
\hline
\end{tabular}

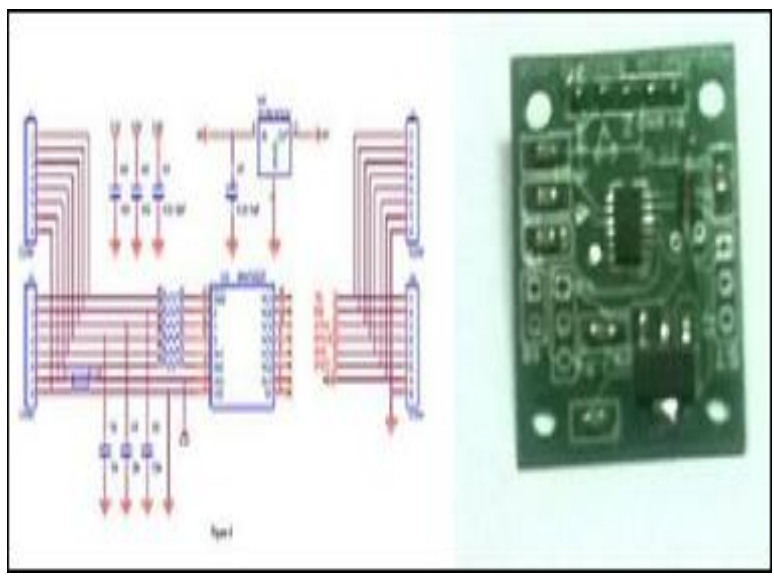

Fig 2.2 MEMS sensor

Published By: 


\section{G. Temperature Sensor:}

In the incubator, light rays and heating pads are used to maintain the warmth of the baby's body. At times, the temperature may rise causing burns and rashes to the baby skins. In order to avoid this, this sensor is used so that the nurse can control the equipment at the need of the hour.

\begin{tabular}{|c|c|c|}
\hline & Normal & Abnormal \\
\hline $\begin{array}{c}\text { Temperature } \\
\text { (analog volts) }\end{array}$ & $36.5^{\circ} \mathrm{C}-37.2^{\circ} \mathrm{C}$ & $\begin{array}{c}\text { If not within the } \\
\text { normal range }\end{array}$ \\
\hline
\end{tabular}

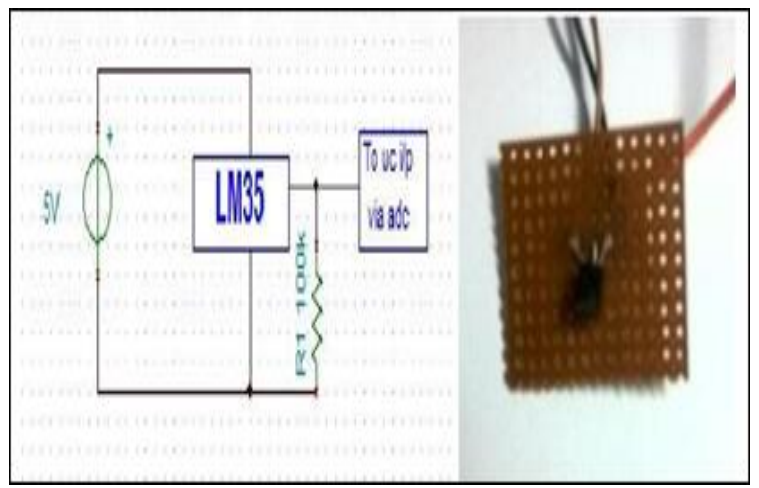

Fig 2.3 Temperature sensor

\section{H. Humidity sensors:}

This sensor is used in the project in order to check up for the dry conditions of the baby skin else it might cause problems to the baby.

Also we can identify the moist conditions within the incubator setup if any. This in a way can reduce the possibility of dangers with presence of water. Thirdly, we can also figure out if there is excess of saliva from the baby's mouth

\begin{tabular}{|c|c|c|}
$\begin{array}{c}\text { Water } \\
\text { content } \\
\text { (ppm) }\end{array}$ & $\begin{array}{c}\text { Output ( } \\
\text { Normal ) }\end{array}$ & Output(Abnormal) \\
\hline 59 & $1.22 \mathrm{~V}$ & $\begin{array}{l}\text { If greater than } \\
1.22(+/- \text { of } 2)\end{array}$ \\
\hline 60 & $1.44 V$ & $\begin{array}{l}\text { If greater than } \\
1.44(+/- \text { of } 2)\end{array}$ \\
\hline
\end{tabular}

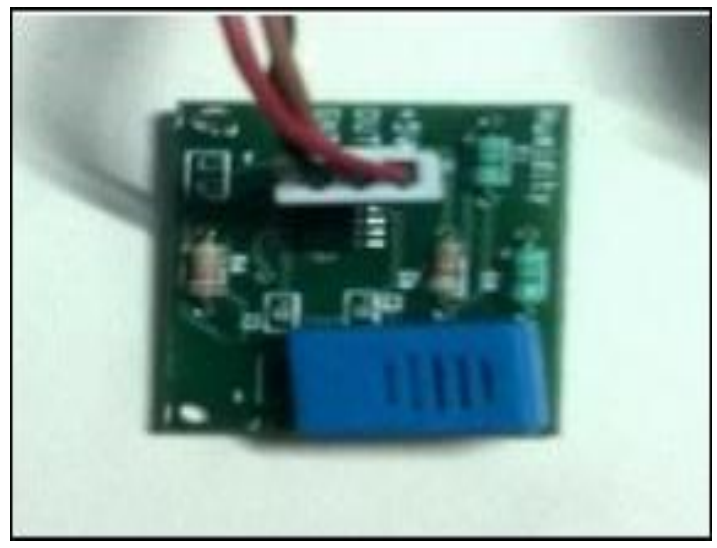

Fig 2.4 Humidity sensor

I. Respirology sensor:
This sensor is mainly used to know if the baby is actually breathing/has normal breathing at sleeping position. We have used this sensor so that it measures the no of pulses corresponding to baby's breath in and out rate.

\begin{tabular}{|l|l|l|}
\hline & Normal & Abnormal \\
\hline $\begin{array}{l}\text { Breath in and } \\
\text { out (once) }\end{array}$ & $\mathbf{0 . 2 5 - 1 . 0 0 ~ V}$ & More than 1V \\
\hline
\end{tabular}

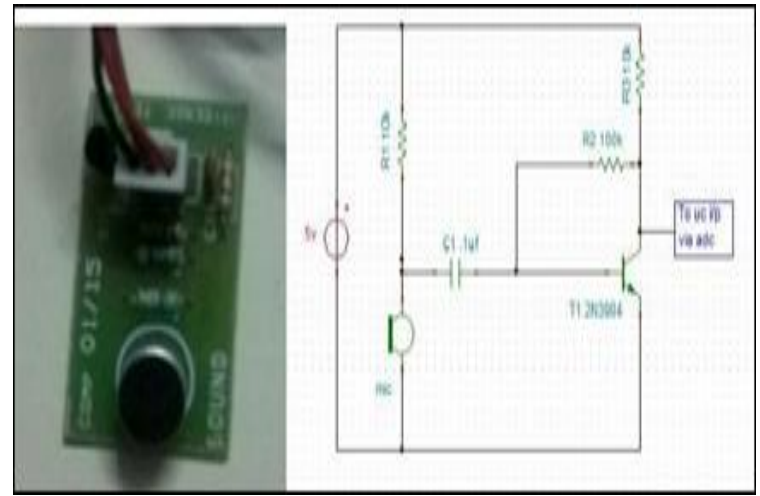

Fig 2.5 Respirology sensor

\section{J. Heartbeat sensor}

When the comparator $\mathrm{o} / \mathrm{p}$ is high ( equal), we get $5 \mathrm{~V}$ digital o/p 0v.So based on the number of high outputs the microcontroller gives us the no of heartbeats per minute

\begin{tabular}{|c|c|c|}
\hline & Normal Rate & $\begin{array}{c}\text { Abnormal } \\
\text { Rate }\end{array}$ \\
\hline New born Baby & $\begin{array}{c}\mathbf{7 0} \text { to } 190 \\
\text { beats per } \\
\text { minute }\end{array}$ & $\begin{array}{c}\text { If not between } \\
\text { the range }\end{array}$ \\
\hline
\end{tabular}

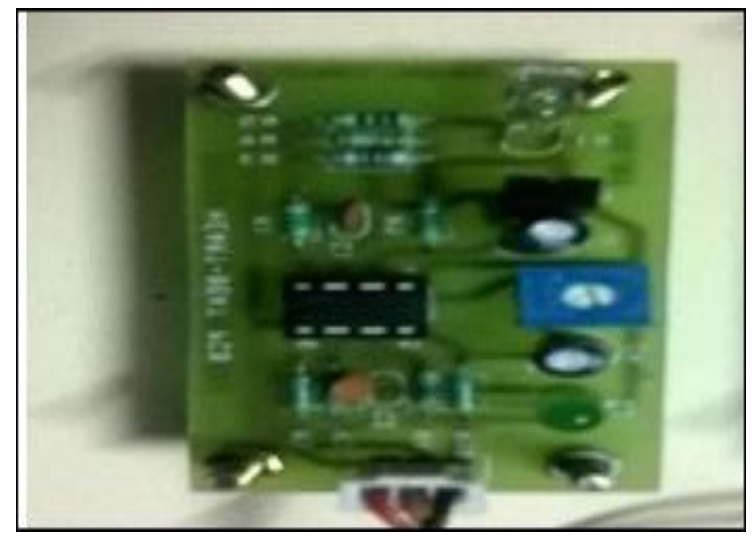

Fig 2.6 Heartbeat sensor

K. Voice Board Module

This module is used to give continuous alert signals in case of any abnormal condition. In our prototype we have recorded three voice messages ( total of $3.2 \mathrm{Mb}$ ) in the channels 1,2and 3( 01,10,11). Push buttons like Play, Record and Playback were used for initial setup.

Runs at +5 volt supply. 


\begin{tabular}{|c|c|}
\hline $\begin{array}{l}\text { CHANNEL } \\
\text { NUMBER }\end{array}$ & MESSAGE \\
\hline 01 & SUFFOCATION WARNING \\
\hline 10 & $\begin{array}{l}\text { CO2 ALERT; EXCEED } \\
\text { THRESHOLD;INCREASE } \\
\text { O2 SUPPLY }\end{array}$ \\
\hline 11 & FITS DETECTED \\
\hline
\end{tabular}

\section{Uart}

A UART, universal asynchronous receiver/transmitter is responsible for performing the main task in serial communications with machines.

The device translates incoming parallel information to serial data which can be transmitted on a communication line.

\section{Zigbee}

ZigBee is a specification for a suite of high level communication protocols using small, low-power digital radios based on the IEEE 802.15.4-2003 standard for wireless personal area networks (WPANs), such as wireless headphones connecting with cell phones via short-range radio communication. The technology specified by the ZigBee specification is designed to be simpler and less costly than other WPANs, such as Bluetooth.

ZigBee is targeted at radio-frequency (RF) applications that need a low data rate, long battery life, and secure networking.

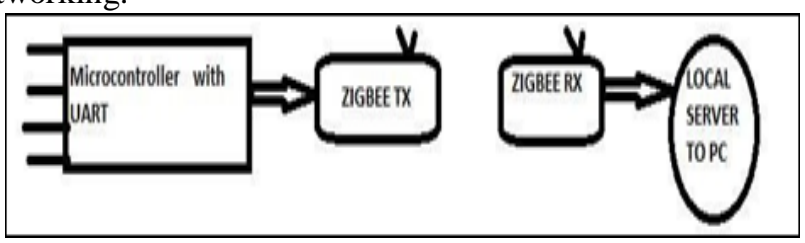

Fig 2.7 Wireless Transmission

\section{N. Relay and motor output module:}

Here the relay is used to turn on the $\mathrm{O} 2$ supply motor valve. When it is realized that there is too much of $\mathrm{CO} 2$, by appropriate programming the motor is signaled with reference to the variable. We use Normally Open Double Pole Double Throw relay with a DC motor

In our model, the relay is given 5Vsupply.

A ULN is present in the circuit to avoid back emf

The relay consists of a pin for external supply of $12 \mathrm{~V}$ to themotor

We used Brushed motor in order to avoid spark generation and noises

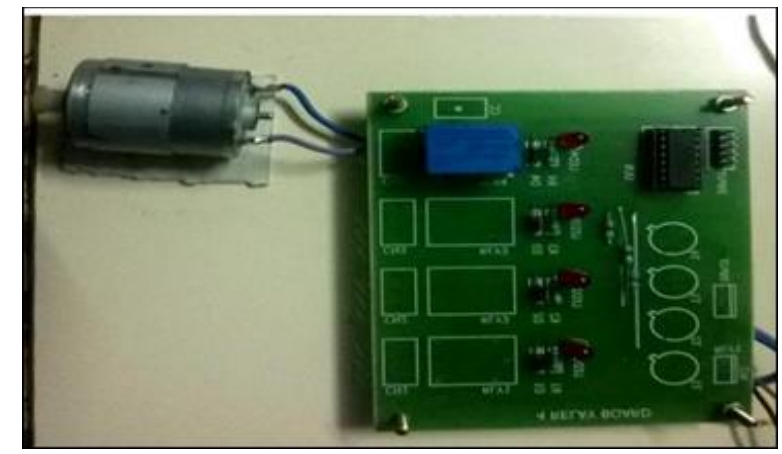

Fig 2.8. Relay with motor for $\mathrm{O} 2$ supply section

\section{SOFTWARE DESCRIPTION}

\section{A. KeiluVision4:}

The new Keil $\mu$ Vision4 IDE has been used since it enhance developer's productivity.

The $\mu$ Vision Debugger from Keil provides a single environment in which we can test, verify, and optimize your application code.

\section{B. Need for Software}

With the help of software programming, the real time parameters of the infant such as temperature, heartbeat and respiratory flow, and humidity, position of the baby and the level of co2 inside the incubator atmosphere can be detected and viewed real time with the help of programming. According to the program fed into the controller the abnormal level of the parameters which are detected are viewed and the alert are given to the concerned efficient. It also helps in keeping the continuous record of the infant and alert is also given during emergency condition.

\section{Algorithm}

Variables are declared for all the sensors used

Bidirectional digital ports ( 0 and 2 ) are used to acquire and give out data

Port pins used for ADC, LCD displays, voice board, relay and motor, transmission section are defined.

Sensor values are digitally converted and checked with their thresholds / Appropriate output devices are initiated

Zigbee receiver constantly checks for the incoming bytes of data

Producing delays and acknowledgment displaying high levels using pushbuttons

\section{PROCESS DESCRIPTION}

\section{A. Problem Definition}

This project aims at proposing an automated system to measure the level of $\mathrm{Co} 2$ in the incubator which when high causes suffocation and also the occurrence of Fits in the baby which are death syndromes and prevent them by immediate actions. Along with this we measure other essential parameters and communicate these details continuously through IoT web technologies

\section{B. Stepwise Procedure}

STEP 1: The power supply is turned on for the process.

STEP 2: All the sensors collect the input Parameter from the baby present in the incubator.

STEP 3: The analog inputs are converted into digital output by $\mathrm{ADC}$ and are loaded into the microcontroller which is processed according to the programming.

STEP 4: when $\mathrm{CO} 2$ level crosses the standard valve, oxygen valve opens.

STEP 5: All the sensed values are displayed.

STEP 6: During abnormal conditions, continuous alerts are played using voiceboard.

STEP 7: All the values from the controller are transmitted 
to ZigBee through UART and wirelessly from there is downloaded to the server.

\section{Process Flow diagram}

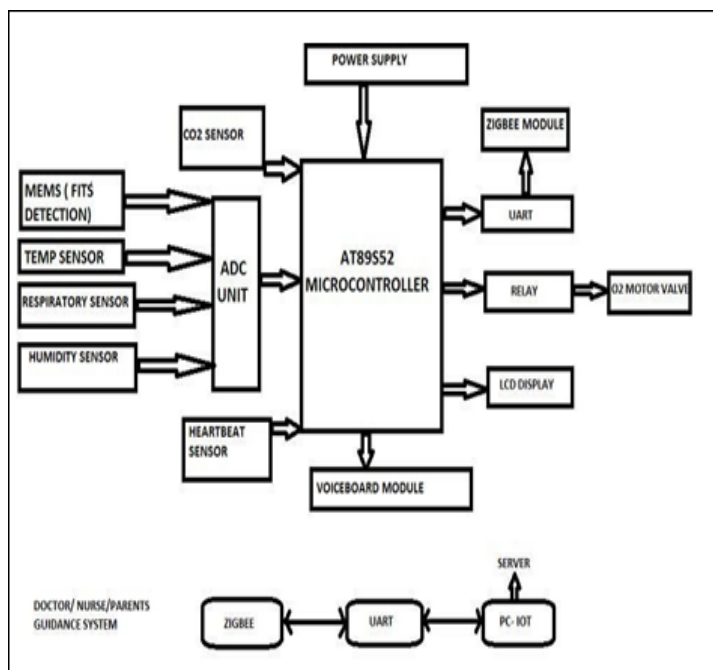

FIG 4.1 Block Diagram Of The Process

In the Input section, all the sensors senses for the $\mathrm{Co} 2$ level, temperature, respiratory rate, heartbeat, humid conditions and for movements within the baby in the incubator. The Sensed values are analog and are converted to digital using ADC whereas o/p from $\mathrm{co} 2$ and heartbeat sensor is sent directly to controller. The converted values are dumped into the microcontroller and are used up for processing and display according to the programming. If within the incubator, co2 does not release properly and if it crosses the threshold, using coding the oxygen valve motor at the $\mathrm{o} / \mathrm{p}$ side is turned out by energizing the relay till normal conditions are met. Likewise every sensed value is displayed on the LCD continuously. During abnormal conditions in any of the above, continuous alerts are played using voice board to the personnel using programming. All the values from the controller are transmitted to ZigBee through UART and wirelessly from there is downloaded to the server. From the local server, continuous recording of measurements are indicated on the webpage to make it globally available to nurses, parents and doctors right from the place wherever they are.

\section{Schematic Diagram Of The Integrated system}

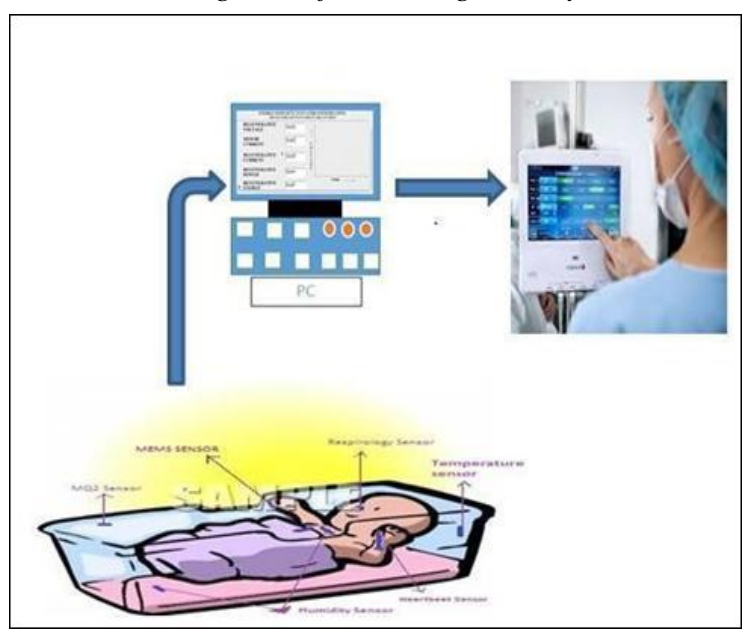

Fig 4.2 Schematic diagram

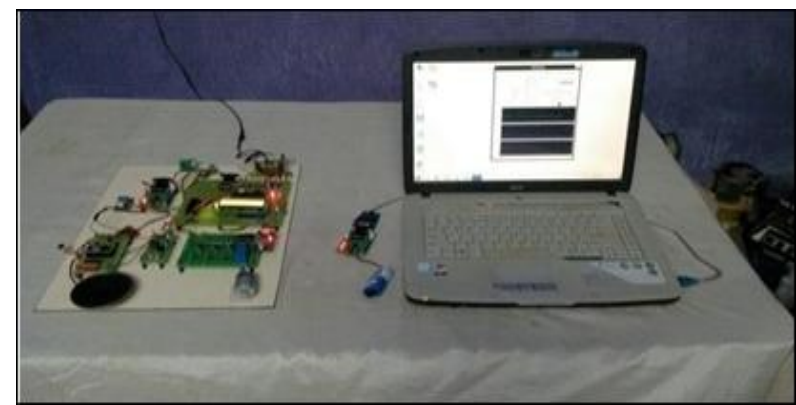

Fig 4.3 Entire setup for demo

\section{RESULTS AND DISCUSSION}

Output during the demo of project would be online viewing of infant's condition from any place while the monitoring is taking place. Immediate alerting mechanism using a speaker is implemented in extreme situations. In case of abnormal $\mathrm{CO} 2$ level in incubator, we can bring back to normal condition via closed loop control. In real time, implementing this wireless safety system would lower the death rate caused by the risks involved with the incubators.

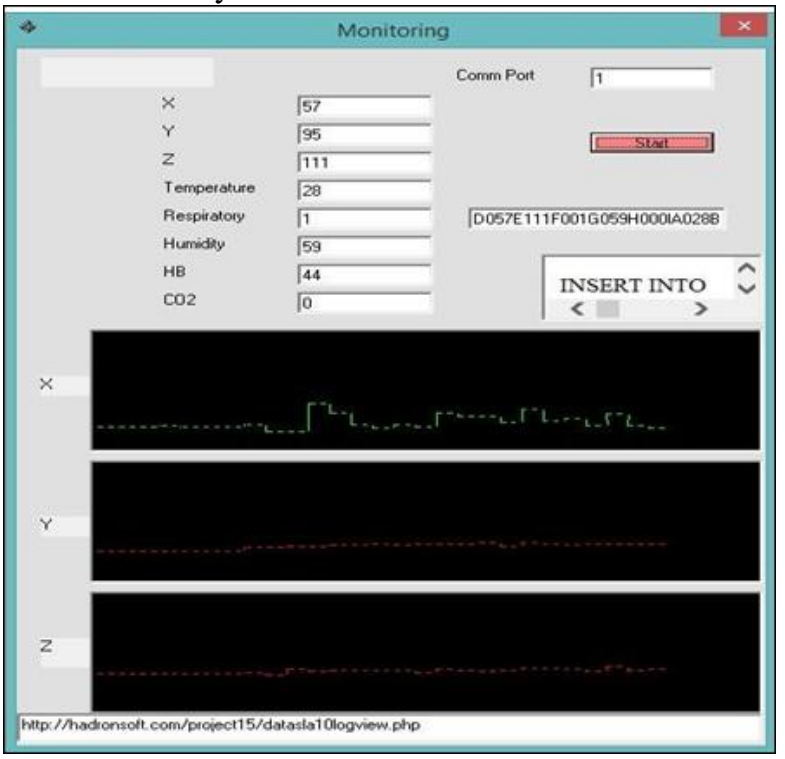

A. Webpage to view $24 * 7$ (IoT)

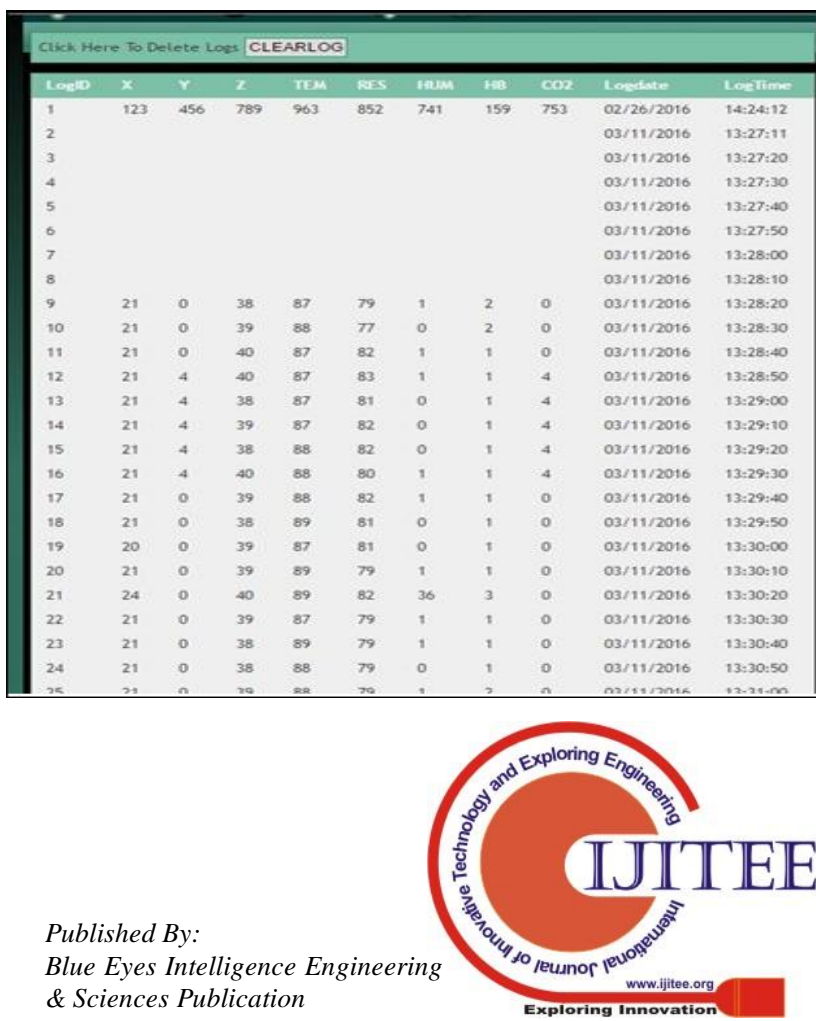




\section{FUTURE WORK}

Our future work is planning to not just use this sensing technology at the Nano level but fabricate all this in a baby fabric cloth which makes it an even easier supervising system. Also we are looking forward to implement better control actions against the risks involved with heating pads etc.

\section{CONCLUSION}

The wireless infant monitoring system is the best method for the prevention of SIDS because it can easily detect and send warning to the parents immediately so that the death rate of infants due to this risk could to decreased to a greater extent

The Wi-Fi is selected as a means of wireless communication because this device is mostly used in hospitals and homes where the Wi-Fi coverage is predominantly available. Wi-Fi can easily connect the device to cloud servers so that a monitoring system is formed without the need of any bridging device. By accessing the cloud service the device can continuously update the health condition of the infant and the device will upload the measured parameters as data to the cloud server in order to prepare a database for future reference.

\section{REFERENCES}

1. F. M. Sullivan And S. M. Barlow, "Review Of Risk Factors For Sudden Infant Death Syndrome (Vol 15, Pg 144, 2001),"Paediatric And Perinatal Epidemiology, Vol. 16, Pp. 96-96,Jan2002.

2. K. Zhang And X. M. Wang, "Maternal Smoking And Increased Risk Of Sudden Infant Death Syndrome: A Meta analysis, "Legal Medicine, Vol. 15, Pp. 115-121, May2013.

3. A.Darwish and A.E Hassanien, "Wearable And Implantable Wireless Sensor Network Solutions For Healthcare Monitoring,"Sensors, Vol. 11, Pp.5561-5595, Jun 2011.

4. J. Ko, C. Y. Lu , M. B. Srivastava, J. A. Stankovic, A. Terzis,And M. Welsh, "Wireless Sensor Networks For Healthcare," Proceedings Of The IEEE, Vol. 98, Pp. 1947- 1960, Nov2010.

5. E. Jovanov, C. C. Y. Poon, G. Z. Yang, And Y. T. Zhang, "Body Sensor Networks: From Theory To Emerging Applications," Ieee Transactions On Information Technology In Biomedicine, Vol. 13, Pp. 859-863, Nov2009.

6. P. C. Jain, "Wireless Body Area Network For Medical Healthcare," IEEE Technical Review, Vol. 28, Pp. $362-$ 371, Jul- Aug2011.

7. G. D. Clifford And D. Clifton, "Wireless Technology In Disease Management And Medicine," Annual Review Of Medicine, Vol 63, Pp.479-492,2012.

8. R. Barry, "Using The Freertos Real Time Kemel Standard Edition",Ed,2010.

\section{AUTHORS PROFILE}

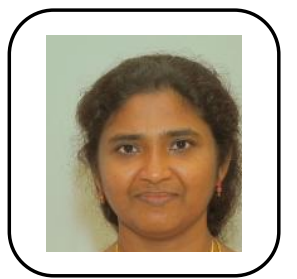

Pearley Stanley was awarded B.E in EIE from Bharathiar university and M.E from Madras University. Her publications are "Development of Control system for Indigenous Electrospinning Machine" Middle-East Journal of Scientific Research, Vol.24, Issue.2. Pg no. 319-324, ISSN 19909233, Jan 2016.
"Development of Control System for indigenous electro spinning machine" TIAR 2016

"Classification of Rice Varieties Using Near Infrared Spectroscopy", TIAR 2015

"Parameter Estimation, Modeling and IMC-PID Control of Temperature using Cascade Control Strategy", NCWTRMC, EEC, Chennai. Pg no. 81-88, Sep 2014

"Fuzzy Logic Based Remote Irrigation system using LabVIEW", NCMCOP, May 2013. Pg no.49-52. 